\title{
Playing Immersive Games on the REVERIE platform
}

\author{
Ioannis Doumanis \\ CTVC Ltd, London, UK \\ ioannis@truetube.co.uk
}

Julie Wall

University of East London (UEL)

The School of Architecture, Computing and Engineering

London, UK

J.Wall@uel.ac.uk

\author{
David S. Monaghan \\ Insight Centre for Data Analytics \\ Dublin City University \\ david.monaghan@dcu.ie
}

\begin{abstract}
REVERIE (REal and Virtual Engagement in Realistic Immersive Environments) [1] is a multimedia and multimodal framework, which supports the creation of immersive games. The framework supports the creation of games integrating technologies such as 3D spatial audio, detection of the player's body movement using Kinect and WIMO sensors, NPCs (Non-Playable Characters) with advanced AI capabilities featuring various levels of representation and gameplay into an immersive 3D environment. A demonstration game was developed for REVERIE, which is an adapted version of the popular Simon Says game. In the REVERIE version, a player tries to follow physical instructions issued by two autonomous agents with different degrees of realism. If a player follows a physical instruction correctly, they are awarded one point. If not, they are deducted one point. This paper presents a technical overview of the game technologies integrated in the Simon Says demo and its evaluation by players with variable computer literacy skills. Finally the potential of REVERIE as an immersive framework for gaming is discussed, followed by recommendations for improvements in future versions of the framework.
\end{abstract}

Keywords- REVERIE framework, Immersive games, NonPlayable Characters (NPCs), Empirical evaluation of games

\section{INTRODUCTION}

A game development framework provides the programming infrastructure to build, deploy and run platform specific or cross-platform games. It can be as simple as a collection of code packed up into a library (e.g., JavaScript [2]) or a more complex system consisted of tools, workflow and supporting a specific set of technologies. Both types of frameworks are designed to help speed up the development process of a game. There are currently several frameworks on the market (e.g., Marmalade [3]) either proprietary or open-source, supporting mainly the development of cross-platform games. Many of these games use design techniques (e.g., strong and interesting narratives and cognitively demanding game tasks) to facilitate immersion into the game (e.g., Bioshock [4]). An immersive gaming experience is synonymous to the "suspension of disbelief" [5], a player feeling as though they are "in" the world that the game creates. For example, having to complete a challenging game task is good for immersion as it ties up the player's mental resources. If the player's mental resources are allocated in completing the task, it is hard for the player to notice all of the game's problems and shortcomings that would otherwise remind them that they are playing a game. Although if done well, these design techniques can result into an immersive gameplay, full player immersion may not always be possible. This is because of the limited range of communication modalities usually supported by these games and the partial completeness of the available sensory information. The former reason refers to the ways by which players communicate with each other and with the game like ways of recognizing the player's physical motion and affect state and match them with in-game action. The latter reason refers to the degree which the available sensory information in a game has the full range of the customary characteristics to create the illusion of immersion. Audio is a good example of sensory information that fails to reproduce the full range of characteristics found in the real world (e.g., direction, location, near, far) to fully immerse players in a gaming experience.

REVERIE (REal and Virtual Engagement in Realistic Immersive Environments) is a framework designed to support full player immersion in game experiences. It achieves this by integrating a wide range of state of the art (SoTa) sensor and AI technologies such as Kinect (v.1 and v.2) [6], wearable sensors, spatial audio, NPCs (Non-Playable Characters) capable of detecting the player's affect state (e.g., head nodes and speech input) as well as a simplified workflow by which games utilising various game design techniques can be implemented. This paper presents an overview of the REVERIE framework with a particular emphasis on its gamerelated technologies and the implementation of Simon Says, an immersive game using the framework. Finally, the evaluation of Simon Says is presented with players of variable computing literacy skills. Based on the evaluation a number of recommendations are made for improvements in future versions of the REVERIE framework. 


\section{THE REVERIE FRAMEWORK}

REVERIE is a software framework developed to support capturing of immersive media, representation, encoding and semi-automated collaborative content production. It is a multiprocess, multi-stream, multi-server, multi-platform and multiuser system (see Figure 1) [7], designed to provide the following functionalities:

- Multimodal and multi sensor signal acquisition

These components (shown as yellow blocks in Figure 1) cover all aspects related to capturing of real world data using various types of sensors and the transformation of this data into suitable types to be further processed, compressed, transmitted visualized and integrated. In particular, they provide: (1) the platforms needed to capture real world objects (mainly moving humans) and scenes using a wide range of sensors (e.g., Clusters of capture cameras, Kinect and wearable sensors); (2) specify a 3D representation format that enables efficient transmission of captured data over the current and future Internet; (3) real-time reconstruction of captured objects/scenes into 3D representations, including geometry and appearance that can be compressed, transmitted, visualized and integrated in virtual volumes with other 3D elements (e.g., avatars and 3D objects); (4) multimodal tracking of users and analysis of user activity; and finally, (5) enable users to generate 3D models of real objects and embed them in virtual scenes from $2 \mathrm{D}$ pictures and videos.

\section{- Networking for immersive communication}

These components (shown as green blocks in Figure 1) realise the underlying network structure needed to deploy robust, scalable and collaborative immersive applications (including immersive games) on the REVERIE framework. The implemented network functionalities can be divided into the following areas: (1) specification of the network structure, components and protocols, (2) specification of a proper naming/addressing scheme for digital objects (e.g.., avatars and other 3D objects) that may exist in the network, (3) design an optimized content caching scheme to support efficient network traffic, (4) development of techniques for compression of 3D video streams, synchronization and caching.

\section{- Interaction and autonomy}

These components (shown as blue blocks in Figure 1) enable the implementation and user interaction with autonomous virtual humans (Non Playable Characters (NPCs)) in immersive applications (including immersive games). In particular, they enable the realization of autonomous virtual humans capable of: (a) coherent and natural behaviours, (b) plausibly interact in virtual spaces, (c) socially interacting with other users in an emotional and expressive manner, (d) interactions with users based on and affected by their emotional state.

\section{- Composition and visualization}

These components (shown as red blocks on Figure 1) enable the composition and visualization of an immersive application (including immersive games) on the REVERIE platform. In particular, they enable: (a) the construction of a virtual environment by specifying the scene structure and navigation system, (b) the visualization of immersive virtual worlds, including existing and imaginary locations, (c) the audiovisual rendering of the virtual worlds, (d) the composition and synchronization of virtual scenes consisted of embedded users, virtual humans and $3 \mathrm{D}$ objects and $2 \mathrm{D}$ artefacts, including the real-time navigation of users within the environment.

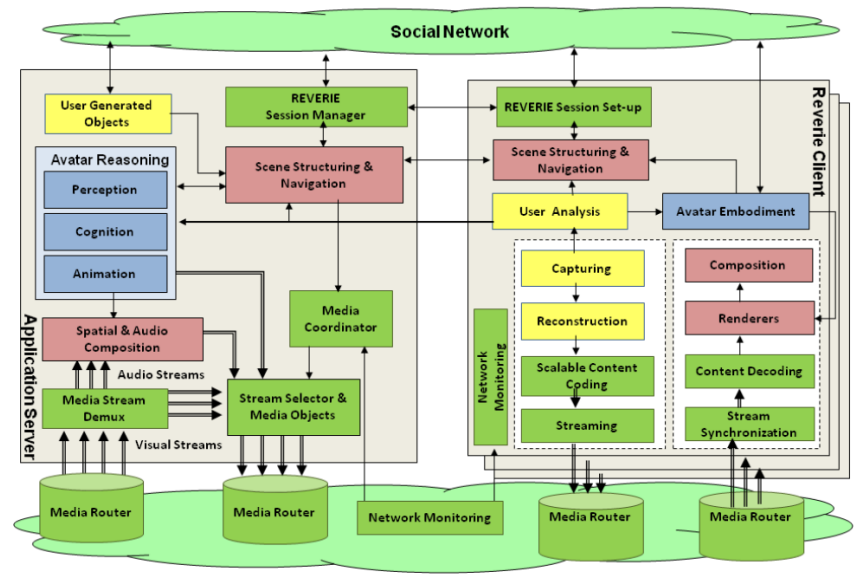

Figure 1. The REVERIE platform architecture

The architecture of the framework makes its use a good solution for the development of immersive games. The social network component can provide integration of social networking in games, though it was not used in the development of the Simon Says game. It supports among other features: (a) a single sign-on system (a) sharing of content created in a REVERIE immersive application (e.g., images and videos) on Facebook (b) an API for integration of the social network with immersive applications developed on the REVERIE framework and others.

\section{DEVELOPMENT OF SIMON SAYS GAME}

The REVERIE framework was used in the development of a version of the well-known Simon Says game. The game supports both single-player and multi-player modes. In this game, participants had to observe the physical instructions issued by two agents (Simon and his assistant). The agents issued physical instructions the one after the other so even if a participant would miss an instruction from Simon, they could still observe it from his assistance. The agents had different degrees of realism, with Simon represented as a standard avatar, while his helper as an HHI Human. If a player followed a physical instruction correctly, they were awarded with one point. If not, they were deducted one point. Also, Simon (and his assistant) sometimes issued physical instructions without using "Simon Says" at the beginning of the instruction. If a player tried to follow the instruction, they were deducted one point. If they did not follow the instruction, they were awarded one point. Points were visualized on the scoreboard of the game. 
Simon is autonomous, there is no player involved in his control system during game play, see Figure 2. Simon gets his knowledge on what to do from an XML script describing how he should behave, what to say when and where, what to ask whom, where to go to, etc. This script has options for flow control, so it can specify a non-linear storyline. However, player input can and does influence execution of the script instructions, e.g. Simon asks the player when they are ready to play the game or whether they want to play again. For this simple dialogue (i.e. agree or disagree) both spoken input and player head movements (nod/shake) are accepted. REVERIE's Speech Recognition functionality supplies information on spoken input and User Affect Analysis functionality supplies head nod/shake input [8].

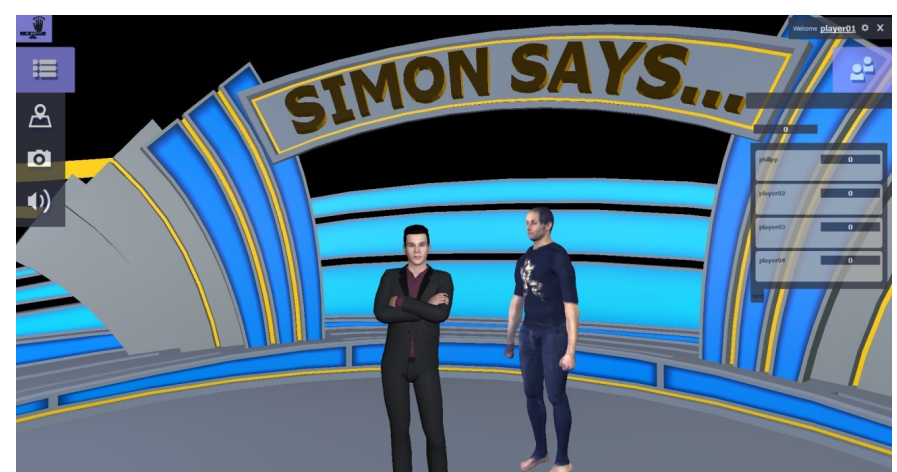

Figure 2. Simon Says environment with the agent "Simon" and his assistant

REVERIE's Reasoning Framework provides the control functionality for Simon [9]. Through this control system, Simon knows about the rules of the game and handles the running of the game autonomously. The agent optionally starts with an explanation of the game and asks if the players are ready to play the game. When the players indicate they are ready, Simon will announce the start and proceed to play the game. Simon selects one from the set of gestures in his repertoire, telling the players which one to perform either with or without the words "Simon says...", performs that gesture himself and then takes some time to analyse what the players are doing. REVERIE's Reasoning Framework gets information from the User Activity Module (which takes input from either the Kinect and/or WIMU devices described below) based on which Simon can award or deduct points. One round of the game repeats this process several times. Simon knows which player won the round and will congratulate this player by addressing that player individually. Next Simon will ask if the players want to continue for another round or stop the game.

The Reasoning Framework can handle several agents simultaneously. Simon has an agent configured to be his assistant, known as the HHI-Human character, see the character on the right in Figure 2. This assistant agent joins Simon in performing the gestures and at the end of a round this assistant agent addresses the winner of the game. The HHI-Human is a hybrid avatar designed to look photo-realistic by combining computer graphics with image based rendering techniques. The photo-realistic appearance is achieved by using texture maps generated from capturing a-priory a real human and updating the texture map on each frame according to the currently desired expression [10]. Gestures as well as expressions have been recorded and pre-processed in a way that each recorded gesture and/or expression can be looped or combined with each other one in random order, see Figure 3. A BML message receiving and interpreting unit has been included in order to allow REVERIE's Reasoning Framework to control the HHI-Human through high-level commands such as nod (for gestures) or attentive (for expressions). Additionally, several audio sequences have been recorded in order to let the HHI-Human speak. Using the spatial audio component, the audio signal is triggered in synchrony with the corresponding facial expression upon the announcement of a winner of a Simon Says game round.

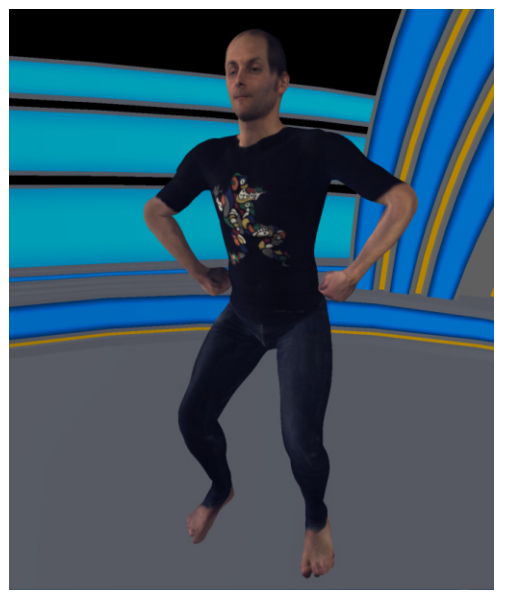

Figure 3. The HHI Human performing a gesture in the Simon Says game

In REVERIE's Simon Says game, the virtual environment reflects a game show studio experience, as represented in Figure 2. A large circular space maximizes the visibility between players while also providing sufficient space for the players to compete with each other and for Simon and his assistant to showcase the physical activities. The autonomous agent, Simon, is placed in the centre of the room near one of the "Simon Says" signs. The HHI-Human assistant is positioned beside the autonomous agent leaving space large enough for 2 to 5 user-controlled avatars to play the game.

Players can communicate with its other and the agents in the game using 3D immersive audio. This type of audio simulates how the sound reverberates in the real world in its way from the source to the listener's ear. This includes reflections from walls, floor and other objects in the virtual scene. The spatial and audio composition module (see Figure 1) uses a spatialisation technique (described in [11]) to simulate 3D audio and reverberation based on the position of current objects in the scene, including animated avatars and on the fly reconstructed humans. This technique, unlike other systems that require multiple speakers and channels, aims at reproducing the $3 \mathrm{D}$ immersive audio by using only two channels. 
The players' physical activity is recognised using low-cost motion capture solutions, utilising Microsoft Kinect and wearable sensors (Wireless/Wearable Inertial Measurement Unit (WIMU)). Activity recognition in the game aims to recognize the gestures and physical actions of players using one of the two sensors or by utilizing a fusion of the sensors for greater accuracy. Gestures enable players to interact with the game GUI (e.g., to increase/decrease the audio volume), while physical actions are integral part of the Simon Says gameplay. For a physical activity to be recognized the full body motion should first be captured and then accurate skeleton and bio-mechanical information should be extracted. The process of extracting an accurate representation of a human skeleton through a Kinect or through body worn sensors is a complex procedure. However, whichever method is utilised to extract the skeleton, the output is still raw motion data and there is not yet any semantic understanding of the data. The process of activity analysis comes into play after an accurate skeleton, or sub-sections of a skeleton, or metadata related to the skeleton, have been extracted.

Several activity recognition techniques were investigated and developed in an attempt to find the most appropriate technique to use in the REVERIE framework. The technique that was chosen to be used as part of the REVERIE platform needed to operate in real-time, show a high accuracy across a variety of different users, i.e. men, women, tall and short etc. There were two main action recognition techniques that were developed as part of the REVERIE project. The first technique was based on the RGB stream to recognise human actions/gestures using multi-view RGB streams. Within [12], we experimented with multi-view 2D features and reconstructed 3D features, namely: 3D Histogram of gradients (HoG3D), Motion History Images (MHI) and Motion History Volume (MHV). Additionally, several popular classifiers and matching algorithms were employed, including Support Vector Machines (SVM), Mahalanobis Distance (MD) and Linear Discriminant Analysis (LDA). However, due to performance restrictions on the CPU during the running of REVERIE platform it was decided not to integrate this approach and to use a more light weight approach utilising the fusion of the Kinect sensor and wearable sensors. This was the second technique, which was based on the Kinect and Wireless/Wearable Inertial Measurement Unit (WIMU) data fusion and which is explained in greater depth in [12]. This technique forms the basis of the activity analysis module in the REVERIE framework that is showcased through the Simon Says game.

TABLE I. TEST RESULTS OF THE GESTURE RECOGNITION FRAMEWORK

\begin{tabular}{|l|l|c|c|c|c|c|}
\hline \multicolumn{1}{|c|}{ Modality } & $\begin{array}{c}\text { Sub.1 } \\
\text { CV \% }\end{array}$ & $\begin{array}{c}\text { Sub.2 } \\
\text { CV\% }\end{array}$ & $\begin{array}{c}\text { Sub.3 } \\
\text { CV \% }\end{array}$ & $\begin{array}{l}\text { Sub.4 } \\
\text { CV \% }\end{array}$ & $\begin{array}{c}\text { Sub.5 } \\
\text { CV \% }\end{array}$ & $\begin{array}{c}\text { All Subj } \\
\text { CV\% }\end{array}$ \\
\hline Kinect & 85.2 & 82.8 & 86.5 & 87.6 & 87.7 & 80.3 \\
\hline ACC & 90.0 & 94.6 & 90.5 & 90.4 & 88.0 & 89.1 \\
\hline K+ACC+Mag & 90.4 & 94.6 & 92.0 & 91.9 & 90.2 & 90.7 \\
\hline
\end{tabular}

A summary of the test results from the REVERIE gesture recognition framework can be seen below where the Accuracy we achieved based on 10 fold cross validation yielded Kinect Only $=80.31 \%$, Acc Only $=89.00 \%$ and Sensor Fusion $=$ $90.67 \%$.

\section{EVALUATION OF SIMON SAYS}

In an empirical evaluation of Simon Says conducted in the laboratory, 31 participants in groups of four had to play the game. All participants were represented as avatars in the game, though the REVERIE framework can support the integration of other form of virtual representations (e.g., onthe-fly-reconstructed 3D humans). At the beginning of each session, an introduction was given to the participants about the context and objectives of the study. Then, participants were given a computer literature survey to fill-in. Once, all participants completed the survey a member of the research team provided training (maximum 10 minutes) on how to play the game. Once participants felt comfortable with the use of the game, they were asked to play two sample activities before playing the main game. Once all participants indicated they felt comfortable with the game, the game was restarted so participants could compete against each other. In the post-task evaluation phase, participants completed a short survey on their experiences with the game and participated in a short interview.

A wide range of subjective and objective data on the players' attitudes (what players say) and behaviors (what players do) with the Simon Says game were collected. The type of data collected and the data collection tools that were used to measure the desired dimensions of the player's experience (i.e., usability, engagement and acceptance) are discussed in [13].

\section{STUDY RESULTS AND DISCUSSION}

\section{Objective Measures}

\section{Completion rate:}

Participants were asked to play the game twice, the first round to practice with a small range of physical motions and the second to play the full game. The completion rate was $100 \%$ in all groups with minimal assistance from the REVERIE researcher team. This shows the effectiveness of the game in enabling participants to complete the assigned game with minimal effort.

Player and system errors:

Player Errors:

- Participants were requested in the introductory training session to make big gestures when trying to follow Simons' physical instructions. However, in the laboratory it was noticed that three out of four participants did not do the physical motion issued by Simon with the required intensity for the game to detect it. As a result, they lost points despite having accurately followed the physical instruction.

System Errors:

- It was noticed in the laboratory that the game occasionally displayed wrong scores on the interface. 
- Simon's assistant (HHI Human) in several occasions failed to accurately repeat a physical instruction

Players' game performance:

To measure if the fusion of the Wireless Inertial Measurement Units (WIMUs) wearable sensors with Kinect made any difference for the participants, the game score of a player wearing WIMUs was compared with the scores of the players in his group each tracked by one Kinect.

TABLE II. SAMPLE PLAYER GAME PERFORMANCE

\begin{tabular}{|l|l|}
\hline \multicolumn{1}{|c|}{ Players } & Total Score \\
\hline Player 1 (WIMUs) & $5 / 7$ \\
\hline Player 2 & $0 / 7$ \\
\hline Player 3 & $3 / 7$ \\
\hline Player 4 & $5 / 7$ \\
\hline
\end{tabular}

It is evident that in terms of game performance the additional sensors did not make any difference. However, it was observed in the laboratory that players wearing the sensors did not put as much effort following the physical instructions issued by Simon as the players using the Kinect sensor.

All WIMU players commented that the sensors were comfortable to wear and play with, which shows that WIMUS can result into improved gameplay without creating any feelings of discomfort to players.

Subjective Assessment

Usability Questionnaire:

Table III, shows the mean ratings for usability, player engagement and player acceptance of the Simon Says game. The low engagement score can be attributed to what participants said in the interviews about Simon not detecting their physical actions correctly.

TABLE III. PLAYER MEAN RATINGS OF THE QUALITY MEASURES IN THE GAME

\begin{tabular}{|c|c|c|}
\hline $\begin{array}{c}\text { Quality } \\
\text { Measured }\end{array}$ & Mean & Std.Dev \\
\hline Usability & 4.6516 & 1.64811 \\
\hline Engagement & 4.3569 & 1.50244 \\
\hline Acceptance & 4.7143 & 1.58881 \\
\hline
\end{tabular}

The one-way ANOVA comparisons taking each questionnaire item as a dependent variable and the participant groups as independent shows a significant main effect of participant groups on Item 10 ("I can learn the commands needed to play in the Simon Says game quite quickly") $(F(6,20)=2.711$; $p<.05$ ) of the Usability Questionnaire (see [16] for the full questionnaire). A post-hoc comparison using Tukey's HSD test [14], on the questionnaire Item 4 ("It is straightforward to learn the commands needed to play in the Simon Says game") using Tukey's HSD test [14], showed that the mean scores of group $06(\mathrm{M}=4.0, \mathrm{SD}=0.81)$ were significantly different from the mean scores of group $01(\mathrm{M}=5.75$, $\mathrm{SD}=$ $0.5)$, group $02(\mathrm{M}=6.0 ., \mathrm{SD}=0.0)$, group $03(\mathrm{M}=5.5, \mathrm{SD}=$ $0.57)$ and group $07(\mathrm{M}=5.2, \mathrm{SD}=0.5)$.
TABLE IV. GROUP MEAN RATINGS FOR ITEM 10 OF THE SimON SAYS QUESTIONNAIRE

\begin{tabular}{|c|c|c|c|c|}
\hline $\begin{array}{c}\text { Player } \\
\text { Groups }\end{array}$ & Mean & $\begin{array}{c}\text { Std. } \\
\text { Deviation }\end{array}$ & $\begin{array}{c}\text { Computer } \\
\text { Literacy } \\
\text { Scores }\end{array}$ & $\begin{array}{c}\text { Std. } \\
\text { Deviation }\end{array}$ \\
\hline Group01 & 5.7500 & .50000 & 2.625 & 1.00 \\
\hline Group02 & 6.0000 & .00000 & 2.312 & 1.25 \\
\hline Group03 & 5.5000 & .57735 & 2.937 & 1.26 \\
\hline Group04 & 5.0000 & 1.41421 & 2.5 & 1.25 \\
\hline Group05 & 5.0000 & 1.00000 & 2.79 & 1.39 \\
\hline Group06 & 4.0000 & .81650 & 3.16 & 1.26 \\
\hline Group07 & 5.2500 & .50000 & 2.375 & 1.12 \\
\hline
\end{tabular}

The particular group (group 06) scored higher in the computer literacy survey (with higher values indicating lower computer literacy skills) which means that they felt the "natural" style of interaction did not benefit them in learning how to play the game in a more efficient way.

Group Interview:

Comments about the two agents (Simon and his assistant):

- The players did not provide a clear answer in terms of realism or the two agents. They generally stated that their representation was realistic enough and they expressed a variety of opinions related to their focus and attention on them.

- Most of the players referred to Simon's assistant as being more distracting in affecting the way they played the game.

- Most of the players agreed that removing Simon's assistant would not make much difference to the experience.

- The players stated that it was difficult for them to follow physical instructions issued by two agents.

- Most of the players could not provide a clear answer to the question which of the two agents was the most engaging one to fully immerse them in the game because they were focused more on Simon who provided the instructions first.

Ways to improve the design of Simon:

1) Most of the players indicated that Simon needs to have higher resolution to look nicer in the game

2) Some players also indicated that more complicated gestures (e.g., wiggle your finger) would make the game more interesting to play.

3) All of the players agreed that audio between the players is necessary to support multiplayer mode.

The results above show overall an above average player acceptance and an average player engagement with the Simon Says game. This is because players, did not follow the issued physical instructions with the required intensity to be recognized by the game. Although in the introductory training session players were requested to make big gestures when following Simon's issued physical instructions, few of the players followed this rule. Then, it was observed in the lab that the increased intensity of physical activity was not a requirement for players wearing the WIMU sensors. In 
addition, participants commented that the sensors were comfortable to wear and play with. Given that the majority of the WIMU participants that were observed were following Simon's physical instruction without "trying too much", it is possible that the fusion of Kinect with WIMUs sensors can provide improved gameplay without causing any feelings of discomfort to players who wear them. Based on the study findings, the following recommendations should be considered for future development of the immersive game Simon Says:

1. Enlarge the score board on the interface to be visible to all players. A score board was available on the interface, but its size was small to be noticed by participants during gameplay.

2. Hide the debug window. The debug window of the game should not be visible to any player and it should be hidden in the background.

3. Remove Simon's assistant from the game as the majority of players said it was merely a distraction.

4. Add an intensity meter on the interface to show the intensity of the player's physical action and the correct intensity that the player should do the action in order to be recognised by the system.

5. Ensure that the interface of the game does not behave unexpectedly (e.g., to display the wrong score)

\section{FUTURE DIRECTIONS}

The first avenue for future work is to make the REVERIE framework compatible with the cloud. Because of this, it was hard to take Simon Says out to the general public and evaluate the player experiences in context (e.g., in the living-room). This factor alone could influence all of the evaluation metrics (either positively or negatively) and provide deeper player insights than those possible in the existing study. Second, as the current study indicated that the fusion of WIMU sensors with Kinect may result to improved gameplay without creating any feeling of discomfort to players it will be interesting to investigate the impact of the fusion of additional sensors (e.g., Lip Motion [17]) with games where detection of complex physical motion is required (e.g., wiggling of fingers). Finally, recent advances in immersive displays (e.g., Oculus Rift [15]) can provide a significant advantage for any games created with the REVERIE framework in terms of entertainment impact. With such displays, players can be immersed deeper in REVERIE gameplay scenarios, which can have a significant impact on how they perceive the game. As previous evaluations of such immersive setups are scarce, the REVERIE research team believes that it is a suitable area where effort should be directed in future development and evaluation work of the REVERIE project.

\section{ACKNOWLEDGMENT}

The research that led to this paper was supported in part by the European Commission under the Contract FP7-ICT-287723 REVERIE.

\section{REFERENCES}

[1] J. Wall. E. Izauierdo. L. Argrvriou. D.S. Monaghan. N.E O'Connor. S. Poulakos. A. Smolic. A Mekuria "REVERIE: Natural human interaction in virtual immersive environments" IN: Proceedings of IEEE International Conference on Image Processing (ICIP 2014), Paris , France, October 27-30, 2014

[2] Collins English Dictionary - Complete \& Unabridged 2012 Digital Edition. William Collins Sons \& Co. 2012. "Javascript". Available at: http://goo.gl/E02nim [Accesed: 21 August 2015]

[3] Marmalade Technologies Ltd: "Marmalade C++ SDK". Available at : https://goo.gl/Ljs3AP [Accessed: 21 August 2015]

[4] Take-Two Interact Software Inc: "BioShock". Available at: https://goo.gl/fUuDCV [Accessed: 21 August 2015]

[5] J. Madigan, "The Psycology of Imemrsion in Video Games". [blog] Available at: http://goo.gl/UTxl89 [Accessed: 21 August 2015]

[6] Microsoft Inc:: Kinect for Windows. Available at: https://goo.gl/Kis41O [Accessed: 21 August 2015]

[7] M. Quacchio, P. Klavdianos, "D2.3 Technical Specification of the REVERIE framework architecture" Available at: http://goo.gl/6GXFpR [Accessed March 11 2015]

[8] F. Kuijk, K.C. Apostolakis, P. Daras, B. Ravenet, H. Wei and D. Monaghan, "Autonomous Agents and Avatars in REVERIE's Virtual Environment', International Conference on 3D Web Technology, Heraklion, Crete, Greece, June 18-21, 2015

[9] F. Kuijk, C. Dareau, B. Ravenet, M. Ochs, K. Apostolakis, P. Daras, D. Monaghan, N.E. O'Connor, J. Wall, E. Izquierdo, S. Van Broeck, 'A Framework for Human-like Behavior in an Immersive Virtual World', International Conference on Digital Signal Processing (DSP), Santorini, Greece, July 1-3, 2013

[10] Fechteler, P., Paier, W., Eisert, P., 'Articulated 3D model tracking with on-the-fly texturing', Image Processing (ICIP), 2014 IEEE International Conference on , Issue Date: $27-30$ Oct. 2014

[11] D.A. Mauro. R. Mekuria. M. Sanna.. "A Binaural Snatialization Svstem for 3D Audio Immersive Communication in Virtual Networked Environments" Proceedings of the Audio Mostly Conference 2013, Pitea, Sweden, September 18-19 2013

[12] M..Gowing, D. Monoghan "D4.3 Report on Developed Tools for multimodal user activity analysis", Available at: http://goo.gl/ASplaX [Accessed: 21 August 2015]

[13] I. Doumanis, "D2.6 Final Report on REVERIE Usability and QoE", Available at : http://goo.gl/7Kufjf [Accessed: 21 August 2015]

[14] Hsu, J (1996): Multiple Comparisons: Theory and Methods, Chapman \& Hall.

[15] Oculus VR LLC: Oculus Rift Virtual Reality Head Mounted Display. Available at: https://goo.gl/PkBE2r [Accessed: 21 August 2015]

[16] I.Doumanis "D3.6 Report on REVERIE field trial", Available at: http://goo.gl/MMcMse [Accessed: 21 August 2015]

[17] Leap Motion Inc: Leap Motion Controller. Aavailable at: https://goo.gl/LDba41 [Accessed: 21 August 2015] 